\title{
ON LIFTING HECKE EIGENFORMS
}

\author{
LYNNE H. WALLING
}

\begin{abstract}
A classical Hilbert modular form $f \in \mathscr{M}_{k}\left(\Gamma_{0}(\mathscr{N}, \mathscr{F}), \chi_{\mathscr{N}}\right)$ cannot be an eigenform for the full Hecke algebra. We develop a means of lifting a classical form to a modular form $F \in \bigoplus_{\lambda} \mathscr{M}_{k}\left(\Gamma_{0}\left(\mathcal{N}, \mathscr{F}_{\lambda}\right), \chi_{\mathscr{N}}\right)$ which is an eigenform for the full Hecke algebra. Using this lift, we develop the newform theory for a space of cusp forms $\mathscr{S}_{k}\left(\Gamma_{0}(\mathscr{N}, \mathscr{F}), \chi_{\mathscr{N}}\right)$; we also use theta series to construct eigenforms for the full Hecke algebra.
\end{abstract}

Since a space $\mathscr{M}_{k}\left(\Gamma_{0}(\mathscr{N}, \mathscr{I}), \chi_{\mathscr{N}}\right)$ of Hilbert modular forms for a fixed weight, character and congruence subgroup is not invariant under the algebra $\mathscr{T}$ of Hecke operators, many authors have enlarged this space of forms to obtain a $\mathscr{T}$-invariant space; in this paper we investigate an alternative approach, restricting our attention to a subalgebra $\mathscr{T}_{0}$ of $\mathscr{T}$ under which the space $\mathscr{M}_{k}\left(\Gamma_{0}(\mathscr{N}, \mathscr{J}), \chi_{\mathscr{N}}\right)$ is invariant. This space is finite-dimensional, so it has a basis consisting of $\mathscr{T}_{0}$-eigenforms; we present here a lifting of such eigenforms to $\mathscr{T}$-eigenforms in the aforementioned larger space, $\mathscr{M}_{k}\left(\mathscr{N}, \chi_{\mathscr{N}}\right)=$ $\bigoplus_{\lambda} \mathscr{M}_{k}\left(\Gamma_{0}\left(\mathscr{N}, \mathscr{J}_{\lambda}\right), \chi_{\mathscr{N}}\right)$. Using this lifting, we are able to develop the newform theory for the space of cusp forms $\mathscr{S}_{k}\left(\Gamma_{0}(\mathscr{N}, \mathscr{I}), \chi_{\mathscr{N}}\right)$. In particular, we show that the subspace $\mathscr{S}_{k}^{+}\left(\Gamma_{0}(\mathscr{N}, \mathscr{I}), \chi_{\mathscr{N}}\right)$ generated by newforms is isomorphic as a $\mathscr{T}_{0}$-module to a subspace of $\mathscr{S}_{k}^{+}\left(\Gamma_{0}(\mathcal{N}, \mathscr{O}), \chi_{\mathscr{N}}\right)$, and that $\mathscr{S}_{k}^{+}\left(\Gamma_{0}(\mathscr{N}, \mathscr{O}), \chi_{\mathscr{N}}\right)$ has a basis of $\mathscr{T}_{0}$-eigenforms which can be lifted to a basis of $\mathscr{T}$-eigenforms of $\mathscr{S}_{k}^{+}\left(\mathscr{N}, \chi_{\mathscr{N}}\right)$. Thus the study of the space of cusp forms $\mathscr{S}_{k}\left(\mathscr{N}, \chi_{\mathscr{N}}\right)$ can be reduced to the study of the more classical space $\mathscr{S}_{k}\left(\Gamma_{0}(\mathscr{N}, \mathscr{O}), \chi_{\mathscr{N}}\right)$. Finally, we extend a classical result, showing that a particular weighted average of theta series attached to lattices is a $\mathscr{T}_{0}$-eigenform, and hence can be lifted to an eigenform for the full Hecke algebra.

The author thanks Winnie Li and John Hsia for helpful conversations, and the referee for a careful reading of this paper.

\section{Preliminaries}

For the most part we follow the definitions of [7]. Let $\mathbf{K}$ be a totally real number field of degree $n$ over $\mathbb{Q}, \mathscr{O}$ its ring of integers, and $\partial$ its different. We define analogs of the group $\Gamma_{0}(N) \subseteq S L_{2}(\mathbb{Z})$ as follows. For $\mathscr{N}$ an integral

Received by the editors November 8, 1989 and, in revised form, March 13, 1990.

1980 Mathematics Subject Classification (1985 Revision). Primary 11F41, 11 E10.

Key words and phrases. Hilbert modular forms, newforms, theta series, quadratic forms. 
ideal of $\mathscr{O}$ and $\mathscr{I}$ a fractional ideal, let

$$
\begin{aligned}
\Gamma_{0}(\mathcal{N}, \mathscr{I}) \\
\quad=\left\{\left(\begin{array}{ll}
a & b \\
c & d
\end{array}\right) \in\left(\begin{array}{cc}
\mathscr{O} & \mathscr{I}^{-1} \partial^{-1} \\
\mathscr{N} \mathcal{S} \partial & \mathscr{O}
\end{array}\right): a d-b c \in \mathscr{O}^{\times}, a d-b c \gg 0\right\}
\end{aligned}
$$

where $a d-b c \gg 0$ means that $a d-b c$ is totally positive. Set $\mathscr{H}=\{\tau \in \mathbb{C}$ : $\operatorname{Im} \tau>0\} ;$ for $f: \mathscr{H}^{n} \rightarrow \mathbb{C}, k=\left(k_{1}, \ldots, k_{n}\right) \in\left(\mathbb{Z}_{+}\right)^{n}$, and $A=\left(\begin{array}{ll}a & b \\ c d\end{array}\right) \in$ $G L_{2}^{+}(\mathbf{K})$ (i.e. $A \in G L_{2}(\mathbf{K})$ with $\operatorname{det} A \gg 0$ ) we set

$$
A \tau=\left(\frac{a^{(1)} \tau_{1}+b^{(1)}}{c^{(1)} \tau_{1}+d^{(1)}}, \ldots, \frac{a^{(n)} \tau_{n}+b^{(n)}}{c^{(n)} \tau_{n}+d^{(n)}}\right)
$$

(where $a^{(j)}$ is the $j$ th conjugate of $a$ ) and

$$
f|A(\tau)=f|_{k} A(\tau)=(\operatorname{det} A)^{k / 2}(c \tau+d)^{-k} f(A \tau) .
$$

We say $f$ is a Hilbert modular form of weight $k$ and character $\chi_{\mathcal{N}}$ with respect to the group $\Gamma_{0}(\mathcal{N}, \mathscr{I})$ if $f: \mathscr{H}^{n} \rightarrow \mathbb{C}$ is an analytic function such that:

(i) $\lim _{\tau \rightarrow i \infty} f \mid A(\tau)$ exists for all $A \in G L_{2}^{+}(\mathbf{K})$, and

(ii) $f \mid A=\chi_{\mathscr{N}}(a) f$ for all $A=\left(\begin{array}{c}a * \\ * *\end{array}\right) \in \Gamma_{0}(\mathscr{N}, \mathscr{I})$;

we say such a function $f$ is a cusp form if the limit in (i) is always 0 . Here $\chi_{\mathscr{N}}$ is a numerical character modulo $\mathscr{N}$ (i.e. $\chi_{\mathscr{N}}:(\mathscr{O} / \mathscr{N})^{\times} \rightarrow \mathbb{C}^{\times}$is a homomorphism); we assume $\chi_{\mathscr{N}}(u)=\operatorname{sgn}(u)^{k}$ for $u \in \mathscr{O}^{\times}$(else only $f=0$ satisfies the above conditions). The collection of all Hilbert modular forms of weight $k$ and character $\chi_{\mathscr{N}}$ with respect to the group $\Gamma_{0}(\mathcal{N}, \mathscr{I})$ constitutes a complex vector space which is denoted by $\mathscr{M}_{k}\left(\Gamma_{0}(\mathscr{N}, \mathscr{I}), \chi_{\mathscr{N}}\right)$; the subspace of cusp forms is denoted by $\mathscr{S}_{k}\left(\Gamma_{0}(\mathscr{N}, \mathscr{I}), \chi_{\mathscr{N}}\right)$.

(In [8], Shimura presents a slightly more general definition of a modular form; although all of the proofs in this paper are valid when we use that definition, we use the more restrictive definition to avoid complicating further our notation.)

Whenever $\mathscr{I}$ and $\mathscr{J}$ are fractional ideals in the same strict class (denoted $\mathscr{I} \sim \mathscr{J})$ then

$$
f \mapsto f \mid\left(\begin{array}{cc}
a & 0 \\
0 & 1
\end{array}\right)
$$

is an isomorphism from $\mathscr{M}_{k}\left(\Gamma_{0}(\mathscr{N}, \mathscr{I}), \chi_{\mathscr{N}}\right)$ onto $\mathscr{M}_{k}\left(\Gamma_{0}(\mathscr{N}, \mathscr{J}), \chi_{\mathscr{N}}\right)$, and from $\mathscr{S}_{k}\left(\Gamma_{0}(\mathscr{N}, \mathscr{I}), \chi_{\mathscr{N}}\right)$ onto $\mathscr{S}_{k}\left(\Gamma_{0}(\mathscr{N}, \mathscr{J}), \chi_{\mathscr{N}}\right)$ (where $a$ here is a totally positive element of $\mathbf{K}$ such that $a \mathscr{I}=\mathscr{J}$ ). This mapping commutes with all the operators we will define on these spaces, thus we will only need one space for each strict ideal class. One of the operators we will define gives us an isomorphism between the spaces $\mathscr{M}_{k}\left(\Gamma_{0}(\mathscr{N}, \mathscr{I}), \chi_{\mathscr{N}}\right)$ and $\mathscr{M}_{k}\left(\Gamma_{0}(\mathcal{N}, \mathcal{J}), \chi_{\mathscr{N}}\right)$ (and between the spaces $\mathscr{S}_{k}\left(\Gamma_{0}(\mathscr{N}, \mathscr{I}), \chi_{\mathscr{N}}\right)$ and $\mathscr{S}_{k}\left(\Gamma_{0}(\mathscr{N}, \mathscr{J}), \chi_{\mathscr{N}}\right)$ ) when $\mathscr{I}$ and $\mathscr{J}$ are in the same complex. (The principal complex consists of all the squares of strict ideal classes; the group of complexes is the strict ideal class group modulo the principal complex. For a more complete discussion of the group of complexes, see $\S 61$ of [2].) Anticipating this, we let $\mathscr{I}_{1}, \ldots, \mathscr{I}_{h^{\prime}}$ 
be fractional ideals such that $\mathscr{F}_{1}^{2}, \ldots, \mathscr{I}_{h^{\prime}}^{2}$ represent the distinct strict ideal classes in the principal complex; so $h^{\prime}$ is the class number and $\mathscr{I}_{1}, \ldots, \mathscr{J}_{h^{\prime}}$ represent the distinct ideal classes. For later convenience, we assume $\mathscr{I}_{1}, \ldots$, $\mathscr{I}_{h^{\prime}}$ are relatively prime to $\mathscr{N}$ (i.e. if $\mathscr{P}$ is a prime dividing $\mathscr{N}$ then $\operatorname{ord}_{\mathscr{P}} \mathscr{I}_{\eta}=$ $0)$. We let $\mathscr{F}_{1}, \ldots, \mathscr{J}_{h / h^{\prime}}$ be fractional ideals which represent the distinct complexes; here $h$ is the strict class number. So the ideals $\mathscr{J}_{\lambda} \mathscr{F}_{\eta}^{2}$ represent all the strict classes. Without loss of generality, we assume $\mathscr{J}_{1} \sim \mathscr{O}$ and $\mathscr{J}_{1}^{2} \sim \mathscr{O}$. Also, we set $\Gamma_{\lambda \eta}(\mathscr{N})=\Gamma_{0}\left(\mathscr{N}, \mathscr{J}_{\lambda} \mathcal{F}_{\eta}^{2}\right)$ and $\Gamma_{\lambda}(\mathcal{N})=\Gamma_{\lambda 1}(\mathscr{N})$.

Let

$$
\mathscr{M}_{k}\left(\mathscr{N}, \chi_{\mathscr{N}}\right)=\bigoplus_{\lambda, \eta} \mathscr{M}_{k}\left(\Gamma_{\lambda \eta}(\mathscr{N}), \chi_{\mathscr{N}}\right),
$$

and let

$$
\mathscr{S}_{k}\left(\mathscr{N}, \chi_{\mathscr{N}}\right)=\bigoplus_{\lambda, \eta} \mathscr{S}_{k}\left(\Gamma_{\lambda \eta}(\mathscr{N}), \chi_{\mathscr{N}}\right)
$$

we say the elements of $\mathscr{M}_{k}\left(\mathscr{N}, \chi_{\mathscr{N}}\right)\left(\right.$ resp. $\left.\mathscr{S}_{k}\left(\mathcal{N}, \chi_{\mathscr{N}}\right)\right)$ are Hilbert modular (resp. cusp) forms of level $\mathscr{N}$.

For the sake of convenience we use Shimura's notation to represent the various operators on $\mathscr{M}_{k}\left(\mathscr{N}, \chi_{\mathscr{N}}\right)$ (see [7]). To use this notation, we consider the forms of $\mathscr{M}_{k}\left(\mathscr{N}, \chi_{\mathscr{N}}\right)$ as functions on $\mathbf{G}_{\mathbf{A}}=G L_{2}\left(\mathbf{K}_{\mathbf{A}}\right)$ where $\mathbf{K}_{\mathbf{A}}$ is the adelization of $\mathbf{K}$. We use $\tilde{a}, \widetilde{b}, \widetilde{c}$ (and so on) to denote elements of $\mathbf{K}_{\mathbf{A}}$. We decompose $\mathbf{G}_{\mathbf{A}}$ as follows. For each $\lambda$ and $\eta$, choose $\tilde{t}_{\lambda}$ and $\tilde{s}_{\eta}$ from $\mathbf{K}_{\mathbf{A}}^{\times}$ such that $\left(\tilde{t}_{\lambda}\right)_{\infty}=1=\left(\tilde{s}_{\eta}\right)_{\infty}, \tilde{t}_{\lambda} \mathscr{O}=\mathscr{J}_{\lambda}$ and $\tilde{s}_{\eta} \mathscr{O}=\mathscr{I}_{\eta} ;$ set

$$
\mathbf{x}_{\lambda \eta}=\left(\begin{array}{cc}
1 & 0 \\
0 & \tilde{t}_{\lambda} \tilde{s}_{\eta}^{2}
\end{array}\right) \text {. }
$$

Let $\mathbf{G}_{\infty+}=\left\{\mathbf{x} \in \mathbf{G}_{\mathbf{A}}\right.$ : $\left.\operatorname{det} \mathbf{x}_{\infty} \gg 0\right\}$ where $\mathbf{x}_{\infty}$ denotes the archimedean part of $\mathbf{x}$, and let $\mathbf{G}_{\mathbb{Q}}$ denote the image of $G L_{2}(\mathbf{K})$ embedded along the diagonal of $\mathbf{G}_{\mathbf{A}}$. For a finite prime $\mathscr{P}$, set

$$
\mathbf{W}_{\mathscr{P}}(\mathscr{N})=\left\{\left(\begin{array}{ll}
a & b \\
c & d
\end{array}\right) \in\left(\begin{array}{cc}
\mathscr{O}_{\mathscr{P}} & \partial^{-1} \mathscr{O}_{\mathscr{P}} \\
\mathscr{N}_{\mathscr{O}} \mathscr{O}_{\mathscr{P}} & \mathscr{O}_{\mathscr{P}}
\end{array}\right): a d-b c \in \mathscr{O}_{\mathscr{P}}^{\times}\right\}
$$

and set $\mathbf{W}(\mathscr{N})=\mathbf{G}_{\infty_{+}} \times \prod_{\mathscr{P}} \mathbf{W}_{\mathscr{P}}(\mathscr{N})$ where the product is over all finite primes $\mathscr{P}$. Then we can wriie $\mathbf{G}_{\mathbf{A}}$ as the disjoint union

$$
\mathbf{G}_{\mathbf{A}}=\bigcup_{\lambda, \eta} \mathbf{G}_{\mathbb{Q}} \mathbf{x}_{\lambda \eta}^{-l} \mathbf{W}(\mathcal{N})
$$

(here $l$ denotes the main involution of $M_{2}(\mathbf{K})$ and its extension to $M_{2}\left(\mathbf{K}_{\mathbf{A}}\right)$ ). For $F \in \mathscr{M}_{k}\left(\mathscr{N}, \chi_{\mathscr{N}}\right)$ and $\mathbf{x}=\alpha \mathbf{x}_{\lambda \eta}^{-l} \mathbf{w} \in \mathbf{G}_{\mathbf{A}}$ (where $\alpha \in \mathbf{G}_{\mathbb{Q}}$ and $\mathbf{w} \in \mathbf{W}(\mathscr{N})$ ) we define

$$
F(\mathbf{x})=\chi_{W}\left(\mathbf{w}^{l}\right)\left(f_{\lambda \eta} \mid \mathbf{w}_{\infty}\right)(\mathbf{i})
$$

where $\mathbf{i}=(i, \ldots, i) \in \mathscr{H}^{n}$ and $\chi_{W}\left(\left(\begin{array}{l}\tilde{a} \tilde{b} \\ \tilde{c} \tilde{d}\end{array}\right)\right)=\chi_{\mathscr{N}}(\tilde{a})=\chi_{\mathscr{N}}(a)$ for $a \in \mathscr{O}$ such that $a \equiv \tilde{a}(\bmod \mathscr{N})$. Note that this definition of $F$ as a function on $\mathbf{G}_{\mathbf{A}}$ is independent of the choices of $\tilde{t}_{\lambda}$ and $\tilde{s}_{\eta}$. 
As discussed in [7], $\mathscr{M}_{k}\left(\mathcal{N}, \chi_{\mathscr{N}}\right)\left(\right.$ resp. $\left.\mathscr{S}_{k}\left(\mathscr{N}, \chi_{\mathscr{N}}\right)\right)$ can be viewed as the space of all functions $F: \mathbf{G}_{\mathbf{A}} \rightarrow \mathbb{C}$ such that:

(1) $F(\alpha \mathbf{x w})=\chi_{W}\left(\mathbf{w}^{l}\right) F(\mathbf{x})$ for all $\alpha \in \mathbf{G}_{\mathbb{Q}}, \quad \mathbf{x} \in \mathbf{G}_{\mathbf{A}}$, and $\mathbf{w} \in \mathbf{W}(\mathscr{N})$ with $\mathbf{w}_{\infty}=1$;

(2) For every pair $\lambda, \eta$, there is a Hilbert modular (resp. cusp) form $f_{\lambda \eta}$ with respect to some group $\Gamma_{0}(\mathscr{M}, \mathscr{I})$ such that $F\left(\mathbf{x}_{\lambda \eta}^{-l} \mathbf{w}_{\infty}\right)=$ $\left(f_{\lambda \eta} \mid \mathbf{w}_{\infty}\right)(\mathbf{i})$ for all $\mathbf{w}_{\infty} \in \mathbf{G}_{\infty+}$.

For $\mathbf{y} \in \mathbf{G}_{\mathbf{A}}$ and $F \in \mathscr{M}_{k}\left(\mathscr{N}, \chi_{\mathscr{N}}\right)$ we define $F \mid \mathbf{y} \in \mathscr{M}_{k}\left(\mathscr{N}, \chi_{\mathscr{N}}\right)$ by

$$
F \mid \mathbf{y}(\mathbf{x})=F\left(\mathbf{x y}^{l}\right)
$$

where $\mathbf{x} \in \mathbf{G}_{\mathbf{A}}$. Clearly $F|\mathbf{y}| \mathbf{y}^{\prime}=F \mid \mathbf{y y}^{\prime}$. Notice that

$$
F\left|\mathbf{y}_{0}\left(\mathbf{x}_{\lambda \eta}^{-l} \mathbf{w}_{\infty}\right)=F\left(\mathbf{x}_{\lambda \eta}^{-l} \mathbf{y}_{0}^{l} \mathbf{w}_{\infty}\right)=F\left(\alpha \mathbf{x}_{\mu \sigma}^{-l} \mathbf{v} \mathbf{w}_{\infty}\right)=\chi_{W}\left(\mathbf{v}^{l}\right)\left(f_{\mu \sigma} \mid \mathbf{v}_{\infty}\right)\right| \mathbf{w}_{\infty}(\mathbf{i})
$$

where $\mathbf{y}_{0}$ denotes the finite part of $\mathbf{y}$ and $\mathbf{x}_{\lambda \eta}^{-l} \mathbf{y}_{0}^{l}=\alpha \mathbf{x}_{\mu \sigma}^{-l} \mathbf{v}$ for some $\alpha \in \mathbf{G}_{\mathbb{Q}}$ and $\mathbf{v} \in \mathbf{W}(\mathscr{N})$ with $\mathbf{v}_{\infty}=\alpha^{-1}$. So if $F: \mathbf{G}_{\mathbf{A}} \rightarrow \mathbb{C}$ satisfies condition (ii), then so does $F \mid \mathbf{y}_{0}$.

For $\mathscr{P}$ a finite prime, $\mathscr{P} \nmid \mathcal{N}$, we define the Hecke operator $T(\mathscr{P})$ on $\mathscr{M}_{k}\left(\mathcal{N}, \chi_{\mathscr{N}}\right)$ by

$$
F \mid T(\mathscr{P})=N(\mathscr{P})^{k_{0} / 2-1}\left(F\left|\left(\begin{array}{cc}
\tilde{p} & 0 \\
0 & 1
\end{array}\right)_{0}+\sum_{b \in \mathscr{O} \mid \mathscr{P}} F\right|\left(\begin{array}{ll}
1 & b \\
0 & \tilde{p}
\end{array}\right)_{0}\right)
$$

where $k_{0}=\max \left\{k_{1}, \ldots, k_{n}\right\}$ and $\tilde{p} \mathscr{O}=\mathscr{P}$; here $N(\mathscr{P})$ denotes the norm of $\mathscr{P}$.

We want the Hecke operators to act on the Fourier coefficients of a Hilbert modular form in the same way the Hecke operators act on those of an elliptic modular form. To obtain such an action, we will need to restrict our attention to Hilbert modular forms which are eigenforms for the operators $S(\mathscr{Q})$, which are defined as follows. For $\mathscr{Q}$ a fractional ideal relatively prime to $\mathscr{N}$, we define the operator $S(\mathscr{Q})$ on $\mathscr{M}_{k}\left(\mathscr{N}, \chi_{\mathscr{N}}\right)$ by

$$
F\left|S(\mathscr{Q})=\bar{\chi}_{\mathscr{N}}(\tilde{q}) F\right|\left(\begin{array}{cc}
\tilde{q} & 0 \\
0 & \tilde{q}
\end{array}\right)_{0}
$$

where $\tilde{q} \mathscr{O}=\mathscr{Q}$. It is easily seen that $S\left(\mathscr{Q}_{1}\right) S\left(\mathscr{Q}_{2}\right)=S\left(\mathscr{Q}_{1} \mathscr{Q}_{2}\right)$.

We let $\mathscr{T}$ denote the algebra generated by the operators $T(\mathscr{P})$ and $S(\mathscr{Q})$; since we can define all Hecke operators $T(\mathscr{I})$ in terms of the operators $T(\mathscr{P})$ and $S(\mathscr{Q})$ (see [7]), we refer to $\mathscr{T}$ as the Hecke algebra.

For $\mathscr{Q}$ an integral ideal we define $B(\mathscr{Q}): \mathscr{M}_{k}\left(\mathcal{N}, \chi_{\mathscr{N}}\right) \rightarrow \mathscr{M}_{k}\left(\mathscr{N} \mathscr{Q}, \chi_{\mathscr{N}}\right)$ by

$$
F\left|B(\mathscr{Q})=N(\mathscr{Q})^{-k_{0} / 2} F\right|\left(\begin{array}{cc}
1 & 0 \\
0 & \tilde{q}^{-1}
\end{array}\right)_{0}
$$

where $\tilde{q} \mathscr{O}=\mathscr{Q}$ (see Proposition 2.3 of [7]). 
Clearly $S(\mathscr{I})$ commutes with $T(\mathscr{P})$ and $B(\mathscr{Q})$; for $\mathscr{P} \nmid \mathscr{N} \mathscr{Q}$, the operators $T(\mathscr{P})$ and $B(\mathscr{Q})$ commute. Notice that for each $\mathbf{y} \in \mathbf{G}_{\mathbf{A}}$ with $\mathbf{y}_{\infty}=1$ and $\tau \in \mathscr{H}^{n}$ we have

$$
\begin{aligned}
(F \mid \mathbf{y})_{\lambda \eta}(\tau) & =(F \mid \mathbf{y})\left(\mathbf{x}_{\lambda \eta}^{-l} \mathbf{w}_{\infty}\right)=F\left(\mathbf{x}_{\lambda \eta}^{-l} \mathbf{y}^{l} \mathbf{w}_{\infty}\right) \\
& =F\left(\alpha \mathbf{x}_{\mu \sigma}^{-l} \mathbf{v} \mathbf{w}_{\infty}\right)=\chi_{W}\left(\mathbf{v}^{l}\right) f_{\mu \sigma} \mid \mathbf{v}_{\infty}(\tau)
\end{aligned}
$$

where $\mathbf{w}_{\infty} \in \mathbf{G}_{\infty+}$ such that $\mathbf{w}_{\infty} \mathbf{i}=\tau, \alpha \in \mathbf{G}_{\mathbb{Q}}$ and $\mathbf{v} \in \mathbf{W}(\mathcal{N})$ with $\mathbf{v}_{\infty}=$ $\alpha^{-1} \in \mathbf{G}_{\mathbb{Q}}$. Thus we can find matrices in $G L_{2}^{+}(\mathbf{K})$ which give the action of an operator on a component space $\mathscr{M}_{k}\left(\Gamma_{\mu \sigma}(\mathscr{N}), \chi_{\mathscr{N}}\right)$; this, however, is awkward since we need different matrices for each pair $\mu, \sigma$.

Sometimes we will restrict these operators to a component space: for a form $f \in \mathscr{M}_{k}\left(\Gamma_{\lambda \eta}(\mathscr{N}), \chi_{\mathscr{N}}\right), \mathbf{y} \in \mathbf{G}_{\mathbf{A}}$ with $\mathbf{y}_{\infty}=1$ and $\tau \in \mathscr{H}^{n}$, set

$$
f \mid \mathbf{y}=(F \mid \mathbf{y})\left(\mathbf{x}_{\mu \sigma}^{-l} \mathbf{w}_{\infty}\right)
$$

where $\mathbf{w}_{\infty} \mathbf{i}=\tau,(\operatorname{det} \mathbf{y}) \mathscr{J}_{\lambda} \mathcal{F}_{\eta}^{2} \sim \mathscr{J}_{\mu} \mathcal{I}_{\sigma}^{2}$, and $F \in \mathscr{M}_{k}\left(\mathscr{N}, \chi_{\mathscr{N}}\right)$ has $f$ as its $\lambda, \eta$-component. Then we see that for $\mathscr{I}_{\eta}^{2} \mathscr{Q}^{2} \sim \mathscr{J}_{\sigma}^{2}$ (with $\mathscr{Q}$ relatively prime to $\mathscr{N}), S(\mathscr{Q})$ defines an isomorphism between the spaces $\mathscr{M}_{k}\left(\Gamma_{\lambda \sigma}(\mathscr{N}), \chi_{\mathscr{N}}\right)$ and $\mathscr{M}_{k}\left(\Gamma_{\lambda \eta}(\mathscr{N}), \chi_{\mathscr{N}}\right)$ (and between $\mathscr{S}_{k}\left(\Gamma_{\lambda \sigma}(\mathscr{N}), \chi_{\mathscr{N}}\right)$ and $\left.\mathscr{S}_{k}\left(\Gamma_{\lambda \eta}(\mathscr{N}), \chi_{\mathscr{N}}\right)\right)$.

We can decompose $\mathscr{M}_{k}\left(\mathscr{N}, \chi_{\mathscr{N}}\right)$ into a direct sum of subspaces, each of which is associated to a Hecke character $\chi$ "extending $\chi_{\mathcal{N}_{\infty}}$ " where $\chi_{\infty}(a)=$ $\operatorname{sgn}(a)^{k}$ and $\chi_{\mathscr{N} \infty}=\chi_{\mathscr{N}} \chi_{\infty}$. (So $\chi$ is a Hecke character such that $\chi\left(\tilde{a}_{\mathscr{N}}\right)=$ $\chi_{\mathscr{N}}(\widetilde{a})$ when $\widetilde{a}$ is a unit at all primes dividing $\mathscr{N}$ and $\widetilde{a}_{\mathcal{N}}$ is the $\mathscr{N}$-part of $\tilde{a}$, and $\chi\left(\tilde{a}_{\infty}\right)=\chi_{\infty}(\tilde{a})$.) For such a Hecke character $\chi$ we set

$$
\mathscr{M}_{k}(\mathcal{N}, \chi)=\left\{F \in \mathscr{M}_{k}\left(\mathcal{N}, \chi_{\mathscr{N}}\right): F(\tilde{s} \mathbf{x})=\chi(\widetilde{s}) F(\mathbf{x}) \text { for all } \tilde{s} \in \mathbf{K}_{\mathbf{A}}^{\times}\right\} .
$$

(Thus $\mathscr{M}_{k}(\mathscr{N}, \chi)$ is an eigenspace for the operators $S(\mathscr{Q})$.) As stated in [7], we have $\mathscr{M}_{k}\left(\mathscr{N}, \chi_{\mathscr{N}}\right)=\bigoplus_{\chi} \mathscr{M}_{k}(\mathscr{N}, \chi)$ where the sum is over all Hecke characters $\chi$ extending $\chi_{\mathcal{N}_{\infty}}$. This can be shown using representation theoretic arguments; we present here a constructive argument. We first show how to construct the Hecke characters $\chi$ which extend $\chi_{\mathcal{N}_{\infty}}$.

Lemma 1.1. There are $h^{\prime}$ Hecke characters $\chi$ extending $\chi_{\mathcal{N}_{\infty}}$ where $h^{\prime}$ is the ideal class number. If $\chi$ and $\chi^{\prime}$ are Hecke characters extending $\chi_{\mathcal{N}_{\infty}}$ then $\bar{\chi} \chi^{\prime}$ is a character on the ideal class group.

Proof. The ideal class group can be decomposed as a product of cyclic subgroups; using Dirichlet's theorem on primes, we let $\mathscr{P}_{1}, \ldots, \mathscr{P}_{r}$ be primes not dividing $\mathscr{N}$ so that cls $\mathscr{P}_{1}, \ldots$, cls $\mathscr{P}_{r}$ generate these cyclic subgroups. Let $\epsilon$ be a character on the ideal class group. For each $j=1, \ldots, r$, let $o\left(\mathscr{P}_{j}\right)$ denote the order of cls $\mathscr{P}_{j}$, and take $p_{j} \in \mathscr{O}$ such that $p_{j} \gg 0$ and $\mathscr{P}_{j}^{o\left(\mathscr{P}_{j}\right)}=p_{j} \mathscr{O}$. Set $\chi^{*}\left(\mathscr{P}_{j}\right)$ equal to $\varepsilon\left(\mathscr{P}_{j}\right)$ times an $o\left(\mathscr{P}_{j}\right)$-root of $\bar{\chi}_{\mathscr{N}_{\infty}}\left(p_{j}\right)$. Then for $\widetilde{s} \in \mathbf{K}_{\mathbf{A}}^{\times}$, we can write $\widetilde{s}=a \tilde{u}$ where $a \in \mathbf{K}^{\times}$and $\tilde{u}$ is a unit at all 
primes dividing $\mathscr{N}$; set

$$
\chi(\widetilde{s})=\chi^{*}\left(\mathscr{P}_{1}\right)^{e_{1}} \cdots \chi^{*}\left(\mathscr{P}_{r}\right)^{e_{r}} \chi_{\mathscr{N} \infty}(\tilde{u})
$$

where $\tilde{u} \mathscr{O}$ is in the class of $\mathscr{P}_{1}^{e_{1}} \ldots \mathscr{P}_{r}^{e_{r}}$. Then $\chi$ is a Hecke character extending $\chi_{\mathcal{N} \infty}$.

Now, suppose $\chi$ and $\chi^{\prime}$ are both Hecke characters extending $\chi_{\mathscr{N} \infty}$. Then $\left(\chi \bar{\chi}^{\prime}\right)^{*}$ is a character on the ideal class group, where $\left(\chi \bar{\chi}^{\prime}\right)^{*}(\operatorname{cls} \mathscr{Q})=\chi(\widetilde{q}) \bar{\chi}^{\prime}(\tilde{q})$ for $\tilde{q} \mathscr{O}=\mathscr{Q}$ relatively prime to $\mathscr{N}$. Thus the Hecke characters exiending $\chi_{\mathscr{N} \infty}$ are in one-to-one correspondence with the characters on the ideal class group.

Notice that if $\chi$ extends $\chi_{\mathscr{N} \infty}$, then $\chi^{*}=\chi \bar{\chi}_{\mathscr{N} \infty}$ is well-defined on ideals relatively prime to $\mathcal{N}$.

Now we show

Proposition 1.2. We have $\mathscr{M}_{k}\left(\mathcal{N}, \chi_{\mathscr{N}}\right)=\bigoplus_{\chi} \mathscr{M}_{k}(\mathcal{N}, \chi)$, and hence we have $\mathscr{S}_{k}\left(\mathcal{N}, \chi_{\mathscr{N}}\right)=\bigoplus_{\chi} \mathscr{S}_{k}(\mathcal{N}, \chi)$ where the sum is over all Hecke characters $\chi$ extending $\chi_{\mathscr{N} \infty}$.

Proof. Clearly $\mathscr{M}_{k}(\mathscr{N}, \chi) \cap \mathscr{M}_{k}\left(\mathscr{N}, \chi^{\prime}\right)=\{0\}$ when $\chi \neq \chi^{\prime}$, so $\bigoplus_{\chi} \mathscr{M}_{k}(\mathscr{N}, \chi)$ $\subseteq \mathscr{M}_{k}\left(\mathscr{N}, \chi_{\mathscr{N}}\right)$. Choose $G=\left(g_{\lambda \eta}\right) \in \mathscr{M}_{k}\left(\mathcal{N}, \chi_{\mathscr{N}}\right)$, and let $\chi_{1}, \ldots, \chi_{h^{\prime}}$ denote the distinct Hecke characters extending $\chi_{\mathscr{N} \infty}$. We know that $\left(\chi_{1} \bar{\chi}_{j}\right)^{*}$ is an ideal class character and $\mathscr{J}_{1}, \ldots, \mathscr{I}_{h^{\prime}}$ are in distinct ideal classes; thus Artin's theorem on the linear independence of characters implies that the matrix

$$
\operatorname{diag}\left(\bar{\chi}_{1}^{*}\left(\mathscr{J}_{1}\right), \ldots, \bar{\chi}_{1}^{*}\left(\mathscr{J}_{h^{\prime}}\right)\right) \cdot\left(\begin{array}{ccc}
\left(\chi_{1} \bar{\chi}_{1}\right)^{*}\left(\mathscr{I}_{1}\right) & \cdots & \left(\chi_{1} \bar{\chi}_{h^{\prime}}\right)^{*}\left(\mathscr{J}_{1}\right) \\
\vdots & \ddots & \vdots \\
\left(\chi_{1} \bar{\chi}_{1}\right)^{*}\left(\mathscr{I}_{h^{\prime}}\right) & \cdots & \left(\chi_{1} \bar{\chi}_{h^{\prime}}\right)^{*}\left(\mathscr{I}_{h^{\prime}}\right)
\end{array}\right)
$$

is invertible. So for each $\lambda$ we can solve the matrix equation

for

$$
\left(\begin{array}{ccc}
\bar{\chi}_{1}^{*}\left(\mathscr{I}_{1}\right) & \cdots & \bar{\chi}_{h^{\prime}}^{*}\left(\mathscr{I}_{1}\right) \\
\vdots & \ddots & \vdots \\
\bar{\chi}_{1}^{*}\left(\mathscr{I}_{h^{\prime}}\right) & \cdots & \bar{\chi}_{h^{\prime}}^{*}\left(\mathscr{I}_{h^{\prime}}\right)
\end{array}\right)\left(\begin{array}{c}
f_{\lambda}^{(1)} \\
\vdots \\
f_{\lambda}^{\left(h^{\prime}\right)}
\end{array}\right)=\left(\begin{array}{c}
g_{\lambda 1} \mid S\left(\mathscr{I}_{1}^{-1}\right) \\
\vdots \\
g_{\lambda h^{\prime}} \mid S\left(\mathscr{I}_{h^{\prime}}^{-1}\right)
\end{array}\right)
$$

$$
f_{\lambda}^{(1)}, \ldots, f_{\lambda}^{\left(h^{\prime}\right)} \in \mathscr{M}_{k}\left(\Gamma_{\lambda}(\mathscr{N}), \chi_{\mathscr{N}}\right) .
$$

(Notice that $g_{\lambda \eta} \mid S\left(\mathscr{J}_{\eta}^{-1}\right) \in \mathscr{M}_{k}\left(\Gamma_{\lambda}(\mathscr{N}), \chi_{\mathscr{N}}\right)$.) Then for each $j$, we set $f_{\lambda \eta}^{(j)}=$ $\bar{\chi}_{j}^{*}\left(\mathscr{I}_{\eta}\right) f_{\lambda}^{(j)} \mid S\left(\mathscr{I}_{\eta}\right)$ and $F^{(j)}=\left(f_{\lambda \eta}^{(j)}\right)$. For any $\eta$ and any fractional ideal $\mathscr{Q}$ relatively prime to $\mathscr{N}$, we have $\mathscr{I}_{\eta}^{2} \mathscr{Q}^{2} \sim \mathscr{I}_{\sigma}^{2}$ for some $\sigma$; thus $\mathscr{I}_{\eta} \mathscr{Q}=a \mathscr{I}_{\sigma}$ for some $a \in \mathbf{K}^{\times}$with $\mathscr{Q}$ relatively prime to $\mathscr{N}$. Then we have

$$
\begin{aligned}
f_{\lambda \eta}^{(j)} \mid S(\mathscr{Q}) & =\bar{\chi}^{*}\left(\mathscr{I}_{\eta}\right) f_{\lambda}^{(j)}\left|S\left(\mathscr{I}_{\eta} \mathscr{Q}\right)=\bar{\chi}^{*}\left(\mathscr{I}_{\eta}\right) f_{\lambda}^{(j)}\right| S\left(a \mathscr{I}_{\sigma}\right) \\
& =\bar{\chi}^{*}\left(\mathscr{I}_{\eta}\right) \chi^{*}\left(\mathscr{I}_{\sigma}\right) f_{\lambda \sigma}^{(j)} \mid S(a \mathscr{O}) \\
& =\chi^{*}\left(a^{-1} \mathscr{Q}\right) f_{\lambda \sigma}^{(j)} \mid S(a \mathscr{O})=\chi^{*}(\mathscr{Q}) f_{\lambda \sigma}^{(j)} .
\end{aligned}
$$

So $F^{(j)} \in \mathscr{M}_{k}\left(\mathscr{N}, \chi_{j}\right)$, and $F^{(1)}+\cdots+F^{\left(h^{\prime}\right)}=G$. 


\section{THE LIFTING}

Let $\mathscr{T}_{0}$ be the subalgebra of the Hecke algebra $\mathscr{T}$ consisting of those mappings in $\mathscr{T}$ which are actually operators on each component space $\mathscr{M}_{k}\left(\Gamma_{\lambda \eta}(\mathscr{N}), \chi_{\mathscr{N}}\right)$. Thus $\mathscr{T}_{0}$ is generated by mappings of the form

$$
T\left(\mathscr{P}_{1}\right)^{r_{1}} \cdots T\left(\mathscr{P}_{s}\right)^{r_{s}} S(\mathscr{Q})
$$

where $r_{j} \in \mathbb{Z}_{\geq 0}$, the $\mathscr{P}_{j}$ are primes ideals not dividing $2 \mathscr{N}$, and $\mathscr{Q}$ is a fractional ideal such that $\mathscr{P}_{1}^{r_{1}} \ldots \mathscr{P}_{s}^{r_{s}} \mathscr{Q}^{2} \sim \mathscr{O}$. To ease the notation, we write $T_{0}\left(\mathscr{P}_{1}^{r_{1}} \ldots \mathscr{P}_{s}^{r_{s}}\right)$ for $T\left(\mathscr{P}_{1}\right)^{r_{1}} \ldots T\left(\mathscr{P}_{s}\right)^{r_{s}}$.

Our goal is to begin with a $\mathscr{T}_{0}$-eigenform $f \in \mathscr{M}_{k}\left(\Gamma_{\lambda \eta}(\mathscr{N}), \chi_{\mathscr{N}}\right)$ and, using its eigenvalues, lift $f$ to a $\mathscr{T}$-eigenform $F \in \mathscr{M}_{k}(\mathscr{N}, \chi)$ (where $\chi$ can be any Hecke character extending $\left.\chi_{\mathcal{N}_{\infty}}\right)$; however, we need to find nonzero eigenvalues to effect this lift. To ease the description of this construction, we make the following

Definition. Let $f \in \mathscr{M}_{k}\left(\Gamma_{\lambda \eta}(\mathscr{N}), \chi_{\mathscr{N}}\right)$ be a $\mathscr{T}_{0}$-eigenform. Let $[\mathscr{I}]$ denote the complex of the strict ideal class of $\mathscr{J}$. (Note that each complex has order 1 or 2.) Set

$$
\mathscr{C}(f)=\left\{[\mathscr{I}]: f \mid T_{0}\left(\mathscr{Q}^{2}\right) S\left(\mathscr{Q}^{-1}\right) \neq 0 \text { for some } \mathscr{Q} \in[\mathscr{I}]\right\}
$$

where it is understood that $\mathscr{Q}$ is an integral ideal relatively prime to $\mathscr{N}$. If we have such an ideal $\mathscr{Q} \in[\mathscr{I}]$ with $f \mid T_{0}\left(\mathscr{Q}^{2}\right) S\left(\mathscr{Q}^{-1}\right) \neq 0$ then we say $\mathscr{Q}$ witnesses $[\mathscr{I}] \in \mathscr{C}(f)$.

Clearly $\mathscr{C}(f)$ is a subgroup of the group of complexes; thus the order of $\mathscr{C}(f)$ is a divisor of $h / h^{\prime}$, which is a power of 2 (see $\S 61$ of [2]).

Theorem 2.1. Suppose $f \in \mathscr{M}_{k}\left(\Gamma_{\lambda \eta}(\mathscr{N}), \chi_{\mathscr{N}}\right)$ is a $\mathscr{T}_{0}$-eigenform such that whenever $[\mathscr{Q}] \notin \mathscr{C}(f)$ we have $f \mid T_{0}(\mathscr{Q})=0$ (where $\mathscr{Q}$ is an integral ideal relatively prime to $2 \mathscr{N})$. Then for any Hecke character $\chi$ extending $\chi_{\mathcal{N}_{\infty}}$, we can construct $|\mathscr{C}(f)| \mathscr{T}$-eigenforms $F \in \mathscr{M}_{k}(\mathcal{N}, \chi)$; if $F$ and $G$ are two of these forms then $G= \pm F_{\varepsilon}$, where $F_{\varepsilon}$ is the twist of $F$ by a character $\varepsilon$ on the group $\mathscr{C}(f)$.

Remark. In Lemma 3.4 we show that any newform satisfies the conditions of the theorem, and in Theorem 4.3 we use theta series to construct forms satisfying these conditions.

Proof. Choose generators $\left[\mathscr{Q}_{1}\right], \ldots,\left[\mathscr{Q}_{s}\right]$ for $\mathscr{C}(f)$ where $\mathscr{Q}_{j}$ witnesses $\left[\mathscr{Q}_{j}\right] \in$ $\mathscr{E}(f)$. For each $j$, choose $c_{j}$ such that

$$
f \mid T_{0}\left(\mathscr{Q}_{j}^{2}\right) S\left(\mathscr{Q}_{j}^{-1}\right)=c_{j}^{2} \bar{\chi}^{*}\left(\mathscr{Q}_{j}\right) f .
$$

Set $f_{\lambda \eta}=f$. If $\left[\mathscr{F}_{\lambda}^{-1} \mathscr{J}_{\mu}\right] \notin \mathscr{C}(f)$ then set $f_{\mu \sigma}=0$ for all $\sigma$. For each $\mu$ such that $\left[\mathscr{J}_{\lambda}^{-1} \mathscr{J}_{\mu}\right] \in \mathscr{C}(f)$, choose $\sigma$ such that $\mathscr{J}_{\mu} \mathscr{I}_{\sigma}^{2} \sim \mathscr{Q}_{1}^{t_{1}} \cdots \mathscr{Q}_{s}^{t_{s}} \mathscr{J}_{\lambda} \mathscr{I}_{\eta}^{2}$ where $t_{j}=0$ or 1 . Set

$$
f_{\mu \sigma}=\frac{1}{c_{1}^{t_{1}} \cdots c_{s}^{t_{s}}} f_{\lambda \eta} \mid T_{0}\left(\mathscr{Q}_{1}^{t_{1}} \cdots \mathscr{Q}_{s}^{t_{s}}\right)
$$


(Note that the exponents $t_{j}$ are uniquely determined by the complex of $\mathscr{F}_{\mu}$.) For each $\sigma^{\prime}$, choose $\mathscr{I}$ relatively prime to $\mathscr{N}$ such that $\mathscr{J}_{\sigma}^{2} \mathscr{J}^{2} \sim \mathscr{J}_{\sigma^{\prime}}^{2}$; set

$$
f_{\mu \sigma^{\prime}}=\bar{\chi}^{*}(\mathscr{I}) f_{\mu \sigma} \mid S(\mathscr{I}) \text {. }
$$

Since $f_{\mu \sigma} \mid S(a \mathscr{O})=\chi^{*}(a \mathscr{O}) f_{\mu \sigma}$ for $a \in \mathbf{K}^{\times}$, the definition of $f_{\mu \sigma^{\prime}}$ is independent of the choice of $\mathscr{I}$.

We claim $F=\left(f_{\mu \sigma^{\prime}}\right)$ is a $\mathscr{T}$-eigenform. Just as in the proof of Proposition $1.2, F \mid S(\mathscr{Q})=\chi^{*}(\mathscr{Q}) F$ for all $\mathscr{Q}$ relatively prime to $\mathscr{N}$. Also, for any $\mu$ and $\sigma^{\prime}$ we have

$$
\begin{aligned}
f_{\mu \sigma^{\prime}} \mid & T_{0}\left(\mathscr{Q}_{j}\right)=\frac{\bar{\chi}^{*}(\mathscr{I})}{c_{1}^{t_{1}} \cdots c_{s}^{t_{s}}} f_{\lambda \eta} \mid T_{0}\left(\mathscr{Q}_{1}^{t_{1}} \cdots \mathscr{Q}_{s}^{t_{s}}\right) T_{0}\left(\mathscr{Q}_{j}\right) S(\mathscr{I}) \\
= & \left\{\begin{array}{l}
\frac{\bar{\chi}^{*}\left(\mathscr{Q}_{j}\right) \bar{\chi}^{*}(\mathscr{I})}{c_{1}^{t_{1}} \cdots c_{s}^{t_{s}}} c_{j}^{2} f_{\lambda \eta} \mid T_{0}\left(\mathscr{Q}_{1}^{t_{1}} \cdots \mathscr{Q}_{j}^{0} \cdots \mathscr{Q}_{s}^{t_{s}}\right) S\left(\mathscr{Q}_{j} \mathscr{I}\right) \quad\left(\text { if } t_{j}=1\right) \\
\frac{\bar{\chi}^{*}(\mathscr{I})}{c_{1}^{t_{1}} \cdots c_{s}^{t_{s}}} f_{\lambda \eta} \mid T_{0}\left(\mathscr{Q}_{j} \mathscr{Q}_{1}^{t_{1}} \cdots \mathscr{Q}_{s}^{t_{s}}\right) S(\mathscr{I}) \quad\left(\text { if } t_{j}=0\right)
\end{array}\right. \\
= & \left\{\begin{array}{l}
c_{j}\left(\frac{\frac{\bar{\chi}}{\bar{\chi}^{*}}\left(\mathscr{Q}_{j} \mathcal{I}\right)}{c_{1}^{t_{1}} \cdots c_{j}^{0} \cdots c_{s}^{t_{s}}} f_{\lambda \eta} \mid T_{0}\left(\mathscr{Q}_{1}^{t_{1}} \cdots \mathscr{Q}_{j}^{0} \cdots \mathscr{Q}_{s}^{t_{s}}\right) S\left(\mathscr{Q}_{j} \mathscr{\mathcal { I }}\right)\right) \quad\left(\text { if } t_{j}=1\right) \\
c_{j}\left(\frac{\bar{\chi}^{*}(\mathscr{I})}{c_{j} c_{1}^{t_{1}} \cdots c_{s}^{t_{s}}} f_{\lambda \eta} \mid T_{0}\left(\mathscr{Q}_{j} \mathscr{Q}_{1}^{t_{1}} \cdots \mathscr{Q}_{s}^{t_{s}}\right) S(\mathscr{I})\right) \quad\left(\text { if } t_{j}=0\right)
\end{array}\right. \\
= & c_{j} f_{\lambda^{\prime} \eta^{\prime}}
\end{aligned}
$$

for appropriate $\lambda^{\prime}$ and $\eta^{\prime}$. Thus $F \mid T_{0}\left(\mathscr{Q}_{j}\right)=c_{j} F$.

Now, choose a prime $\mathscr{P} \nmid \mathscr{N}$; if $[\mathscr{P}] \notin \mathscr{C}(f)$ then $f \mid T(\mathscr{P})=0$ and by our constuction, $F \mid T(\mathscr{P})=0$. Suppose $[\mathscr{P}] \in \mathscr{C}(f)$. Then $\mathscr{P} \sim \mathscr{Q}_{1}^{-r_{1}} \ldots \mathscr{Q}_{s}^{-r_{s}} \mathscr{J}^{-2}$ where $r_{j}=0$ or 1 and $\mathscr{I}$ is relatively prime to $\mathscr{N}$. Then $T_{0}\left(\mathscr{R} \mathscr{Q}_{1}^{r_{1}} \cdots \mathscr{Q}_{s}^{r_{s}}\right) S(\mathscr{J})$ $\in \mathscr{T}_{0}$ and $\mathscr{T}$ is a commutative algebra, so remembering how we defined $f_{\mu \sigma^{\prime}}$ we see that

$$
f_{\mu \sigma^{\prime}} \mid T_{0}\left(\mathscr{P} \mathscr{Q}_{1}^{r_{1}} \cdots \mathscr{Q}_{s}^{r_{s}}\right) S(\mathscr{J})=c f_{\mu \sigma^{\prime}}
$$

for some constant $c$ which is independent of $\mu$ and $\sigma^{\prime}$. So

$$
c F=F\left|T_{0}\left(\mathscr{P} \mathscr{Q}_{1}^{r_{1}} \cdots \mathscr{Q}_{s}^{r_{s}}\right) S(\mathscr{J})=\chi^{*}(\mathscr{J}) c_{1}^{r_{1}} \cdots c_{s}^{r_{s}} F\right| T(\mathscr{P})
$$

and hence $F \mid T(\mathscr{P})=c \bar{\chi}^{*}(\mathscr{J}) F / c_{1}^{r_{1}} \cdots c_{s}^{r_{s}}$. Thus $\mathrm{F}$ is a $\mathscr{T}$-eigenform.

Suppose we choose a set of generators $\left[\mathscr{Q}_{j}^{\prime}\right]$ of $\mathscr{C}(f)$ where $\mathscr{Q}_{j}^{\prime}$ is a witness; then $F \mid T_{0}\left(\mathscr{Q}_{j}^{\prime}\right)=c_{j}^{\prime} F$ for some $c_{j}^{\prime} \neq 0$, and $f \mid T_{0}\left(\mathscr{Q}_{j}^{\prime 2}\right) S\left(\mathscr{Q}_{j}^{\prime-1}\right)=c_{j}^{\prime 2} \bar{\chi}^{*}\left(\mathscr{Q}_{j}^{\prime}\right) f$. The form constructed using $f, \mathscr{Q}_{j}^{\prime}$ and $c_{j}^{\prime}$ gives us $F$, and hence regardless of the choices for the generators of $\mathscr{C}(f)$ and their representative witnesses, we obtain the same $|\mathscr{C}(f)|$ lifts of $f$.

Now, let $F$ be one of the forms constructed above and let $\varepsilon$ be a character on $\mathscr{C}(f)$. Extend $\varepsilon$ so that $\varepsilon\left(\left[\mathscr{J}_{\mu}\right]\right)=0$ for $\left[\mathscr{F}_{\mu}\right] \notin \mathscr{C}(f)$. We define $F_{\varepsilon}$ by

$$
F_{\varepsilon}(\mathbf{x})=\varepsilon([\operatorname{det}(\mathbf{x})]) F(\mathbf{x}) \text {. }
$$


So if $F$ is the lift of $f$ using $\mathscr{Q}_{j}$ and $c_{j}$, then $\pm F_{\varepsilon}$ is the lift of $f$ using $\mathscr{Q}_{j}$ and $\varepsilon\left(\left[\mathscr{Q}_{j}\right]\right) \cdot c_{j}$. Unless $\varepsilon=1, F \neq \pm F_{\varepsilon}$. Thus the lifts of $f$ to $\mathscr{M}_{k}(\mathscr{N}, \chi)$ are (up to sign) twists of each other.

Remark. If $F \in \mathscr{M}_{k}(\mathscr{N}, \chi)$ and $G \in \mathscr{M}_{k}\left(\mathscr{N}, \chi^{\prime}\right)$ are lifts of $f$ where $\chi \neq \chi^{\prime}$, then $F$ and $G$ are linearly independent.

\section{NEWFORMS}

To define a newform, we first observe that we can define an inner product on $\mathscr{S}_{k}\left(\mathscr{N}, \chi_{\mathscr{N}}\right):$ for $F, G \in \mathscr{S}_{k}\left(\mathscr{N}, \chi_{\mathscr{N}}\right)$,

$$
\langle F, G\rangle=\sum_{\lambda, \eta}\left\langle f_{\lambda \eta}, g_{\lambda \eta}\right\rangle
$$

where $\left\langle f_{\lambda \eta}, g_{\lambda \eta}\right\rangle$ is the Petersson inner product (see (2.27) of [7]). With this inner product, $\bigoplus_{\lambda, \eta} \mathscr{S}_{k}\left(\Gamma_{\lambda \eta}(\mathcal{N}), \chi_{\mathscr{N}}\right)$ becomes an orthogonal sum. As Shimura shows, the operators $T(\mathscr{P})$ and $S(\mathscr{Q})$ are (essentially) Hermitian on each space $\mathscr{S}_{k}(\mathscr{N}, \chi)$ (see Proposition 2.4 of [7]). We define $\mathscr{S}_{k}^{-}\left(\mathscr{N}, \chi_{\mathscr{N}}\right)$ to be the subspace of $\mathscr{S}_{k}\left(\mathcal{N}, \chi_{\mathscr{N}}\right)$ which is generated by the forms $F \mid B(\mathscr{Q})$ where $F$ is a form of a lower level (i.e. of a level $\mathscr{N}^{\prime}$ where $\mathscr{N}^{\prime}$ is a proper divisor of $\mathscr{N}$ ); we set

$$
\mathscr{S}_{k}^{-}(\mathscr{N}, \chi)=\mathscr{S}_{k}(\mathscr{N}, \chi) \cap \mathscr{S}_{k}^{-}\left(\mathscr{N}, \chi_{\mathscr{N}}\right) \text {. }
$$

We define $\mathscr{S}_{k}^{+}\left(\mathscr{N}, \chi_{\mathscr{N}}\right)$ to be the orthogonal complement of $\mathscr{S}_{k}^{-}\left(\mathscr{N}, \chi_{\mathscr{N}}\right)$, and we set

$$
\mathscr{S}_{k}^{+}(\mathscr{N}, \chi)=\mathscr{S}_{k}(\mathscr{N}, \chi) \cap \mathscr{S}_{k}^{+}\left(\mathscr{N}, \chi_{\mathscr{N}}\right) .
$$

Similarly, for each $\lambda$ and $\eta$ we define $\mathscr{S}_{k}^{-}\left(\Gamma_{\lambda \eta}(\mathscr{N}), \chi_{\mathscr{N}}\right)$ to be the subspace of $\mathscr{S}_{k}\left(\Gamma_{\lambda \eta}(\mathscr{N}), \chi_{\mathscr{N}}\right)$ which is generated by $f \mid B(\mathscr{Q}) \in \mathscr{S}_{k}\left(\Gamma_{\lambda \eta}(\mathscr{N}), \chi_{\mathscr{N}}\right)$ where $f$ is a form of a lower level, and we define

$$
\mathscr{S}_{k}^{+}\left(\Gamma_{\lambda \eta}(\mathscr{N}), \chi_{\mathscr{N}}\right) \subseteq \mathscr{S}_{k}\left(\Gamma_{\lambda \eta}(\mathscr{N}), \chi_{\mathscr{N}}\right)
$$

to be the orthogonal complement of $\mathscr{S}_{k}^{-}\left(\Gamma_{\lambda \eta}(\mathscr{N}), \chi_{\mathscr{N}}\right)$. Then, the arguments of [3] (cf. [6]) extend in a straightforward manner, giving us multiplicity-one in $\mathscr{S}_{k}^{+}(\mathscr{N}, \chi)$ :

Theorem 3.1. Let $\chi$ be a Hecke character extending $\chi_{\mathcal{N}_{\infty}}$. Then $\mathscr{S}_{k}^{+}(\mathscr{N}, \chi)$ has a basis of simultaneous eigenforms for the Hecke operators $T(\mathscr{P})$; if two such forms have the same eigenvalues for all but a finite number of the operators $T(\mathscr{P})$ (where $\mathscr{P}$ denotes a prime ideal not dividing $2 \mathscr{N}$ ), then the forms are linearly dependent.

Corollary 3.2. $\mathscr{S}_{k}^{+}\left(\mathscr{N}, \chi_{\mathscr{N}}\right)$ has a basis $\mathscr{T}$-eigenforms.

Now we consider the component spaces $\mathscr{S}_{k}\left(\Gamma_{\lambda \eta}(\mathscr{N}), \chi_{\mathscr{N}}\right)$. 
Lemma 3.3. The projection map $F=\left(f_{\lambda \eta}\right) \mapsto\left(f_{\lambda 1}\right)$ takes $\mathscr{S}_{k}^{-}(\mathscr{N}, \chi)$ onto $\bigoplus_{\lambda} \mathscr{S}_{k}^{-}\left(\Gamma_{\lambda}(\mathscr{N}), \chi_{\mathscr{N}}\right)$ and hence takes $\mathscr{S}_{k}^{+}(\mathscr{N}, \chi)$ onto $\bigoplus_{\lambda} \mathscr{S}_{k}^{+}\left(\Gamma_{\lambda}(\mathscr{N}), \chi_{\mathscr{N}}\right)$. Proof. Clearly $\operatorname{proj}(F \mid B(\mathscr{Q})) \in \bigoplus_{\lambda} \mathscr{S}_{k}^{-}\left(\Gamma_{\lambda}(\mathscr{N}), \chi_{\mathscr{N}}\right.$ ) (where the level of $F$ is a divisor of $\left.\mathscr{N} \mathscr{Q}^{-1}\right)$. For each $\lambda$, choose $g_{\lambda}=f_{\mu \eta} \mid B\left(\mathscr{Q}_{\lambda}\right) \in \mathscr{S}_{k}^{-}\left(\Gamma_{\lambda}(\mathscr{N}), \chi_{\mathscr{N}}\right)$ where $f_{\mu \eta} \in \mathscr{S}_{k}\left(\Gamma_{\mu \eta}\left(\mathscr{N}^{\prime}\right), \chi_{\mathscr{N}}\right)$ and $\mathscr{Q}_{\lambda} \mathscr{N}^{\prime} \mid \mathcal{N}$; set

$$
f_{\mu \sigma}=\chi^{*}\left(\mathscr{F}_{\eta}\right) \bar{\chi}^{*}\left(\mathscr{I}_{\sigma}\right) f_{\mu \eta} \mid S\left(\mathscr{I}_{\eta}^{-1} \mathscr{F}_{\sigma}\right)
$$

and take $F_{\lambda}$ to be the element of $\mathscr{S}_{k}\left(\mathcal{N} / \mathscr{Q}_{\lambda}, \chi\right)$ whose $\mu, \sigma$-component is $f_{\mu \sigma}$ and whose other components are 0 . Then $G=\sum_{\lambda} F_{\lambda} \mid B\left(\mathscr{Q}_{\lambda}\right) \in \mathscr{S}_{k}^{-}(\mathscr{N}, \chi)$, and $\operatorname{proj} G=\left(g_{\lambda}\right)$. Since $\operatorname{proj}\left(G+G^{\prime}\right)=\operatorname{proj} G+\operatorname{proj} G^{\prime}$, it follows that proj is a surjective map.

Suppose $G \in \mathscr{S}_{k}^{-}(\mathscr{N}, \chi)$ and $\langle F, G\rangle=0$. Since

$$
\begin{aligned}
\left\langle f_{\lambda \eta}, g_{\lambda \eta}\right\rangle & =\left\langle f_{\lambda \sigma}\left|S\left(\mathscr{I}_{\eta} \mathscr{I}_{\sigma}^{-1}\right), g_{\lambda \sigma}\right| S\left(\mathscr{I}_{\eta} \mathcal{I}_{\sigma}^{-1}\right)\right\rangle \\
& =\left\langle f_{\lambda \sigma}\left|A, g_{\lambda \sigma}\right| A\right\rangle=\left\langle f_{\lambda \sigma}, g_{\lambda \sigma}\right\rangle
\end{aligned}
$$

where $A \in G L_{2}^{+}(\mathbf{K})$ gives the action of $S\left(\mathscr{J}_{\eta} \mathcal{F}_{\sigma}^{-1}\right)$, we have

$$
\langle\operatorname{proj} F, \operatorname{proj} G\rangle=\frac{1}{h^{\prime}}\langle F, G\rangle=0
$$

and thus

$$
\operatorname{proj}: \mathscr{S}_{k}^{+}(\mathscr{N}, \chi) \longrightarrow \bigoplus_{\lambda} \mathscr{S}_{k}^{+}\left(\Gamma_{\lambda}(\mathscr{N}), \chi_{\mathscr{N}}\right) .
$$

We know $\operatorname{proj}\left(\mathscr{S}_{k}^{+}(\mathscr{N}, \chi) \oplus \mathscr{S}_{k}^{-}(\mathscr{N}, \chi)\right)=\bigoplus_{\lambda} \mathscr{S}_{k}\left(\Gamma_{\lambda}(\mathscr{N}), \chi_{\mathscr{N}}\right)$, so

$$
\operatorname{proj}\left(\mathscr{S}_{k}^{+}(\mathscr{N}, \chi)\right)=\bigoplus_{\lambda} \mathscr{S}_{k}^{+}\left(\Gamma_{\lambda}(\mathscr{N}), \chi_{\mathscr{N}}\right) .
$$

Lemma 3.4. Let $f \in \mathscr{S}_{k}^{+}\left(\Gamma_{\lambda \eta}(\mathcal{N}), \chi_{\mathscr{N}}\right)$ be a $\mathscr{T}_{0}$-eigenform. If $[\mathscr{Q}] \notin \mathscr{C}(f)$ then $f \mid T_{0}(\mathscr{Q})=0$. Hence we can lift $f$ to $|\mathscr{C}(f)|$ linearly independent $\mathscr{T}$-eigenforms in $\mathscr{S}_{k}^{+}(\mathscr{N}, \chi)$; these lifts of $f$ are (up to sign) twists of each other.

Proof. First we suppose that $f \mid T_{0}\left(\mathscr{Q}^{2}\right) S\left(\mathscr{Q}^{-1}\right)=0$; we show that $f \mid T_{0}(\mathscr{Q})=0$. A basis of $\mathscr{T}$-eigenforms for $\mathscr{S}_{k}^{+}(\mathscr{N}, \chi)$ projects to a spanning set of $\mathscr{T}_{0}^{-}$ eigenforms for $\mathscr{S}_{k}^{+}\left(\Gamma_{\lambda \eta}(\mathscr{N}), \chi_{\mathscr{N}}\right)$, thus we have $f=f_{1}+\cdots+f_{m}$ where each $f_{j}$ is nonzero and is the projection of some $\mathscr{T}$-eigenform $F_{j} \in \mathscr{S}_{k}^{+}(\mathscr{N}, \chi)$. Now, this means that

$$
F_{j} \mid T_{0}\left(\mathscr{Q}^{2}\right) S\left(\mathscr{Q}^{-1}\right)=0
$$

and since $F_{j}$ is a $\mathscr{T}$-eigenform, $F_{j} \mid T_{0}(\mathscr{Q})=0$. (Recall that $\mathscr{T}$ is a commutative algebra and $F_{j} \mid S\left(\mathscr{Q}^{-1}\right)=\bar{\chi}^{*}(\mathscr{Q}) F_{j}$.) Thus

$$
f\left|T_{0}(\mathscr{Q})=f_{1}\right| T_{0}(\mathscr{Q})+\cdots+f_{m} \mid T_{0}(\mathscr{Q})=0,
$$


and so by Theorem 2.1 we can lift $f$ to $|\mathscr{C}(f)|$ linearly independent $\mathscr{T}$ eigenforms $F$ in $\mathscr{S}_{k}(\mathscr{N}, \chi)$.

We now show that a lift $F=\left(f_{\mu \sigma}\right)$ of $f$ is a newform. For

$$
g_{\mu \sigma} \in \mathscr{S}_{k}^{-}\left(\Gamma_{\mu \sigma}(\mathcal{N}), \chi_{\mathscr{N}}\right)
$$

we have

$$
\left\langle f_{\mu \sigma}, g_{\mu \sigma}\right\rangle=\left\langle c f_{\lambda \eta} \mid T_{0}(\mathscr{Q}) S(\mathscr{I}), g_{\mu \sigma}\right\rangle
$$

the operators $S(\mathscr{I})$ and $T_{0}(\mathscr{Q})$ are (essentially) Hermitian, and they map oldforms to oldforms (see [7] and [6]). Thus $\left\langle f_{\mu \sigma}, g_{\mu \sigma}\right\rangle=0$ and hence $F \in$ $\mathscr{S}_{k}^{+}(\mathcal{N}, \chi)$.

Now we show that multiplicity-one in the product space $\mathscr{S}_{k}^{+}(\mathscr{N}, \chi)$ implies that multiplicity-one (with regard to the algebra $\mathscr{T}_{0}$ ) holds in each component space.

Theorem 3.5. If $f, g \in \mathscr{S}_{k}^{+}\left(\Gamma_{\lambda \eta}(\mathcal{N}), \chi_{\mathscr{N}}\right)$ are $\mathscr{T}_{0}$-eigenforms with the same eigenvalues, then $f$ and $g$ are linearly dependent. Furthermore, $\mathscr{S}_{k}^{+}\left(\Gamma_{\lambda \eta}(\mathscr{N}), \chi_{\mathscr{N}}\right)$ is isomorphic (as a $\mathscr{T}_{0}$-module) to a subspace of

$$
\mathscr{S}_{k}^{+}\left(\Gamma_{1}(\mathscr{N}), \chi_{\mathscr{N}}\right)=\mathscr{S}_{k}^{+}\left(\Gamma_{0}(\mathscr{N}, \mathscr{O}), \chi_{\mathscr{N}}\right) .
$$

Proof. The mapping $f \mapsto f \mid S\left(\mathscr{I}_{\eta}^{-1}\right)$ is a $\mathscr{T}_{0}$-module isomorphism, thus we may assume that $f, g \in \mathscr{S}_{k}^{+}\left(\Gamma_{\lambda}(\mathscr{N}), \chi_{\mathscr{N}}\right)$. By Lemma 3.4, we see that we can use $f$ and $g$ to construct $\mathscr{T}$-eigenforms in $\mathscr{S}_{k}^{+}(\mathscr{N}, \chi)$. We see by the construction of these $\mathscr{T}$-eigenforms that we can construct $F$ and $G$ to have the same eigenvalues for $\mathscr{T}$ with $f$ as the $\lambda, 1$-component of $F$, and $g$ as that of $G$. Thus by multiplicity-one for $\mathscr{S}_{k}^{+}(\mathscr{N}, \chi), F$ and $G$ are linearly dependent and hence $f$ and $g$ must be linearly dependent as well. Furthermore, since $F$ is a $\mathscr{T}$-eigenform in $\mathscr{S}_{k}^{+}(\mathscr{N}, \chi)$, its 1,1 -component $f_{1}$ must be nonzero (see [6]). Thus $f_{1} \in \mathscr{S}_{k}^{+}\left(\Gamma_{1}(\mathscr{N}), \chi_{\mathscr{N}}\right)$ is a $\mathscr{T}_{0}$-eigenform with the same eigenvalues as $f \in \mathscr{S}_{k}^{+}\left(\Gamma_{\lambda}(\mathscr{N}), \chi_{\mathscr{N}}\right)$. (In fact, we see from the construction of $F$ that $f=c f_{1} \mid T_{0}(\mathscr{Q}) S(\mathscr{F})$ where $\mathscr{Q}$ is a witness for [Q⿻ $] \subseteq \mathscr{C}(f)$, $\mathscr{J}_{\lambda} \mathscr{Q} \mathscr{J}^{2} \sim \mathscr{O}$, and $c$ is some nonzero constant.) So a basis of $\mathscr{T}_{0}$-eigenforms for $\mathscr{S}_{k}^{+}\left(\Gamma_{\lambda}(\mathscr{N}), \chi_{\mathscr{N}}\right)$ corresponds to a linearly independent set of $\mathscr{T}_{0}$-eigenforms in $\mathscr{S}_{k}^{+}\left(\Gamma_{1}(\mathscr{N}), \chi_{\mathscr{N}}\right)$; since $\mathscr{T}$ is a commutative algebra, this correspondence preserves the $\mathscr{T}_{0}$-eigenvalues, meaning that if $f_{\lambda} \in \mathscr{S}_{k}^{+}\left(\Gamma_{\lambda}(\mathscr{N}), \chi_{\mathscr{N}}\right)$ corresponds to $f_{1} \in \mathscr{S}_{k}^{+}\left(\Gamma_{1}(\mathscr{N}), \chi_{\mathscr{N}}\right)$ then $f_{\lambda}$ and $f_{1}$ have the same eigenvalues for all operators in $\mathscr{T}_{0}$. Thus as a $\mathscr{T}_{0}$-module, $\mathscr{S}_{k}^{+}\left(\Gamma_{\lambda}(\mathscr{N}), \chi_{\mathscr{N}}\right)$ is isomorphic to a subspace of $\mathscr{S}_{k}^{+}\left(\Gamma_{1}(\mathscr{N}), \chi_{\mathscr{N}}\right)$.

Notice that the dimension of $\mathscr{P}_{k}^{+}\left(\Gamma_{\lambda}(\mathscr{N}), \chi_{\mathscr{N}}\right)$ is equal to the number of subgroups $\mathscr{C}\left(f^{(j)}\right)$ which contain $\left[\mathscr{J}_{\lambda}\right]$ where $\left\{f^{(1)}, \ldots, f^{(t)}\right\}$ is a basis of for 
$\mathscr{S}_{k}^{+}\left(\Gamma_{1}(\mathscr{N}), \chi_{\mathscr{N}}\right)$. Thus we get

Corollary 3.6. For $\mathscr{S}_{k}^{+}\left(\Gamma_{1}(\mathscr{N}), \chi_{\mathscr{N}}\right)$, let $\left\{f^{(1)}, \ldots, f^{(t)}\right\}$ be a basis of $\mathscr{T}_{0}^{-}$ eigenforms then

$$
\operatorname{dim} \mathscr{S}_{k}^{+}(\mathscr{N}, \chi)=\sum_{j}\left|\mathscr{C}\left(f^{(j)}\right)\right| \leq \frac{h}{h^{\prime}} \cdot \operatorname{dim} \mathscr{S}_{k}^{+}\left(\Gamma_{1}(\mathscr{N}), \chi_{\mathscr{N}}\right) .
$$

Proposition 1.2 and Theorem 3.1 show that we have multiplicity-one in $\mathscr{S}_{k}\left(\mathscr{N}, \chi_{\mathscr{N}}\right)$ when we consider all operators in the algebra $\mathscr{T}$. The Hecke operators $T(\mathscr{P})$ (where $\mathscr{P}$ is a prime ideal) generate a subalgebra of $\mathscr{T}$; the multiplicity of eigenvalues in $\mathscr{S}_{k}^{+}\left(\mathscr{N}, \chi_{\mathscr{N}}\right)$ for this subalgebra depends in part on "how often" we have $F \mid T(\mathscr{P})=0$. To allow us to describe this more precisely, we have the

Definition. Let $f \in \mathscr{S}_{k}\left(\Gamma_{\lambda}(\mathscr{N}), \chi_{\mathscr{N}}\right)$ be a $\mathscr{T}_{0}$-eigenform; set

$$
\mathscr{L}(f)=\left\{\operatorname{cls} \mathscr{I}: f \mid T_{0}(\mathscr{Q}) \neq 0 \text { for some } \mathscr{Q} \in \operatorname{cls} \mathscr{I}\right\}
$$

where $\operatorname{cls} \mathscr{I}$ denotes the ideal class of $\mathscr{I}$, and $\mathscr{Q}$ is understood to be an integral ideal relatively prime to $\mathscr{N}$.

Then, extending Theorem 5 of [3], we have

Theorem 3.7. Let $F \in \mathscr{S}_{k}^{+}\left(\mathscr{N}, \chi_{\mathscr{N}}\right)$ be a $\mathscr{T}$-eigenform; let $f$ denote the 1,1 component of $F$. There are exactly $h^{\prime}(f)=h^{\prime} /|\mathscr{L}(f)|$ linearly independent forms $G \in \mathscr{S}_{k}^{+}\left(\mathcal{N}, \chi_{\mathscr{N}}\right)$ such that the 1,1-component of $G$ is $f$ and $G$ has the same eigenvalues as $F$ under all the Hecke operators $T(\mathscr{P}$ ) (where $\mathscr{P}$ is a prime ideal not dividing $2 \mathscr{N}$ ). We can obtain some of these forms $G$ by twisting $F$ by characters on the ideal class group modulo $\mathscr{L}(f)$; the number of distinct twists of $F$ obtained in this way is $h^{\prime}(f)$ divided by the number of ideal classes whose squares lie in $\mathscr{L}(f)$.

Proof. Since $F$ is a $\mathscr{T}$-eigenform, $F \in \mathscr{S}_{k}(\mathscr{N}, \chi)$ for some Hecke character $\chi$ extending $\chi_{\mathscr{N} \infty}$. For $\mathscr{P}$ a prime not dividing $\mathscr{N}$, we know that

$$
F \mid T(\mathscr{P})= \pm \sqrt{\chi^{*}(\mathscr{P}) c_{\mathscr{P}}} F
$$

where $f \mid T(\mathscr{P})^{2} S\left(\mathscr{P}^{-1}\right)=c_{\mathscr{P}} f$. Suppose we have $G \in \mathscr{P}_{k}^{+}\left(\mathscr{N}, \chi^{\prime}\right)$ a $\mathscr{T}$ eigenform with 1,1-component $f$; then

$$
G \mid T(\mathscr{P})= \pm \sqrt{\chi^{* *}(\mathscr{P}) c_{\mathscr{P}}} G .
$$

If $F$ and $G$ have the same eigenvalues for all $T(\mathscr{P})$, then we must have $\left(\bar{\chi} \chi^{\prime}\right)^{*}(\mathscr{P})=1$ whenever $c_{\mathscr{P}} \neq 0$.

On the other hand, if $\chi^{\prime}$ extends $\chi_{\mathscr{N}_{\infty}}$ with $\left(\bar{\chi} \chi^{\prime}\right)^{*}(\mathscr{P})=1$ whenever $c_{\mathscr{P}} \neq$ 0 , then we can use $f$ to construct $G \in \mathscr{S}_{k}^{+}\left(\mathscr{N}, \chi^{\prime}\right)$ such that $G$ is a $\mathscr{T}$. eigenform with the same eigenvalues as $F$ for all the $T(\mathscr{P})$.

We know that $\left(\bar{\chi} \chi^{\prime}\right)^{*}$ is a character on the ideal class group; there are $h^{\prime} /|\mathscr{L}(f)|$ characters which are trivial on the subgroup $\mathscr{L}(f)$. Thus there 
are $h^{\prime} /|\mathscr{L}(f)|$ Hecke characters $\chi^{\prime}$ extending $\chi_{\mathscr{N} \infty}$ such that $\left(\bar{\chi} \chi^{\prime}\right)^{*}(\mathscr{P})=1$ whenever $c_{\mathscr{P}} \neq 0$; for each such $\chi^{\prime}$ we can use $f$ to construct exactly one form $G \in \mathscr{S}_{k}^{+}\left(\mathcal{N}, \chi^{\prime}\right)$ such that $G$ is a $\mathscr{T}$-eigenform with the same eigenvalues as $F$ for all the Hecke operators. We know $\mathscr{S}_{k}^{+}\left(\mathscr{N}, \chi_{\mathscr{N}}\right)$ has a basis of $\mathscr{T}$-eigenforms, thus the first assertion of the theorem holds.

To prove the second assertion, let $\varepsilon$ be a character on the ideal class group modulo $\mathscr{L}(f)$; consider $\varepsilon$ as a character on ideals relatively prime to $\mathscr{N}$. Then we can view $\varepsilon$ as a Hecke character with conductor $\mathscr{O}$; as shown in [6], $F_{\varepsilon} \in \mathscr{S}_{k}^{+}\left(\mathscr{N}, \chi \varepsilon^{2}\right)$ (where $F_{\varepsilon}$ is defined by $\left.F_{\varepsilon}(\mathbf{x})=\varepsilon(\operatorname{det} \mathbf{x}) F(\mathbf{x})\right)$. Clearly $F$ and $F_{\varepsilon}$ have the same eigenvalues for $T(\mathscr{P})$. Unless $\varepsilon^{2}=1, \mathscr{S}_{k}^{+}(\mathscr{N}, \chi) \cap$ $\mathscr{S}_{k}^{+}\left(\mathscr{N}, \chi \varepsilon^{2}\right)=\{0\}$; thus $F=F_{\varepsilon}$ when $\varepsilon^{2}=1$, and $F$ and $F_{\varepsilon}$ are linearly independent otherwise.

\section{THETA SERIES}

Let $V$ be a quadratic space of even dimension $2 k$ over the field $\mathbf{K}$ with a totally positive quadratic form $Q$; let $L$ be a lattice on $V$, and let $L^{\#}$ denote the dual of $L$ (see [5]). As shown in [11] (see also [1]), the theta series attached to $L$

$$
\theta(L, \tau)=\sum_{x \in L} e^{\pi i \operatorname{Tr}(Q(x) \tau)}
$$

is a modular form with a quadratic character $\chi_{L}$ and weight $k$ for the group

$$
\Gamma_{0}^{1}(S(L), N(L))=\left\{A \in \Gamma_{0}(S(L), N(L)): \operatorname{det} A=1\right\},
$$

where $N(L)$, the norm of $L$, is the fractional ideal generated by the set $\left\{\frac{1}{2} Q(x): x \in L\right\}$, and $S(L)$, the level (or stufe) of $L$, is the product of $N(L)^{-1} N\left(L^{\#}\right)^{-1}$ and perhaps some dyadic primes (see [11] for a precise definition). Note that a nondyadic prime $\mathscr{P}$ divides $S(L)$ if and only if $L_{\mathscr{P}}=$ $L \otimes \mathscr{O}_{\mathscr{P}}$ is not modular (where $\mathscr{O}_{\mathscr{P}}$ is the localization of $\mathscr{O}$ at $\mathscr{P}$ ). Also, we see that we can relax the conditions in [11] to extend the transformation formula (2), giving us the transformation formula

$$
\theta\left(L, \frac{a \tau+b}{c \tau+d}\right)=(c+d \tau)^{k} \tau^{k} d^{-k} \sum_{x \in L / d L} e\left(\frac{b}{d} Q(x)\right) \theta(L, \tau)
$$

where $\left(\begin{array}{ll}a & b \\ c & d\end{array}\right)$ is any matrix in $\Gamma_{0}^{1}(S(L), N(L))$. (The crucial observation to make when extending this formula is that with such a matrix, local considerations show that $b L \cap d \widetilde{L}=b d L$ and thus for any $x \in \widetilde{L}, b x_{0}+x$ runs over $\tilde{L} / d \tilde{L}$ as $x_{0}$ runs over $L / d L$.) With this transformation formula, the arguments used to prove Theorem 3.7 of [11] show that $\theta(L, \tau)$ is a modular form of weight $k$ for the group $\Gamma_{0}^{1}(S(L), N(L))$ with character $\chi_{L}$, and $\chi_{L}$ is trivial if and only if $L / \mathscr{P}_{L}$ is hyperbolic for all primes $\mathscr{P}$ not dividing $2 S(L)$. 
We define the weighted average of the theta series attached to the lattices in the genus of $L$, gen $L$, to be

$$
\theta(\operatorname{gen} L, \tau)=\sum_{L_{j}} \frac{1}{\left|O\left(L_{j}\right)\right|} \theta\left(L_{j}, \tau\right)
$$

where the sum runs over a complete set of representatives $L_{j}$ of the isometry classes in gen $L$, and $O\left(L_{j}\right)$ denotes the orthogonal group of $L_{j}$. As is fairly clear, $\theta(\operatorname{gen} L, \tau)$ is independent of the choices of representatives $L_{j}$.

Siegel showed that $\theta(\operatorname{gen} L, \tau)$ is an Eisenstein series (see [9-10]); for $\mathbf{K}=$ $\mathbb{Q}$, it is well known that the space spanned by Eisenstein series has a basis of eigenforms for the Hecke operators (see Chapter IV of [4]). Our goal is to use a weighted average of forms $\theta(\operatorname{gen} L, \tau)$ to produce a $\mathscr{T}$-eigenform.

To examine the action of $\mathscr{T}_{0}$ on $\theta(\operatorname{gen} L, \tau)$, we first state a consequence of Theorem 7.3 and Proposition 6.1 of [11]:

Theorem 4.1. If $\mathscr{P}$ is a prime ideal not dividing $2 S(L)$ and $L / \mathscr{P} L$ is hyperbolic, then

$$
\theta(L, \tau) \mid T(\mathscr{P})=N(\mathscr{P})^{k / 2} \lambda^{-1} \sum_{K} \theta(K, \tau)
$$

where $\lambda=\left(N(\mathscr{P})^{k-2}+1\right) \cdots\left(N(\mathscr{P})^{0}+1\right)$ and the sum is taken over all $\mathscr{P}$ sublattices $K$ of $L$, i.e. over all sublattices $K$ of $L$ such that $\mathscr{P} L \subseteq K$ and $K / \mathscr{P} L$ is a maximal tc:ally isotropic subspace of $L / \mathscr{P} L$. Furthermore, each $\theta(K, \tau)$ is a modular form with character $\chi_{L}$ and weight $k$ for the group $\Gamma_{0}^{1}(S(L), \mathscr{P} N(L))$.

Remark. Note that the operators used in [11] are the same as those defined by Eichler; in our current notation, Eichler's operator $V(\mathscr{Q})$ is the operator $S\left(\mathscr{Q}^{-1}\right)$, and Eichler's operator $T(\mathscr{P})$ is $N(\mathscr{P})^{k_{0} / 2} T(\mathscr{P}) S\left(\mathscr{P}^{-1}\right.$ ) (see [1] or [11]). Note also that we may need to follow Eichler's operators by the isomorphism $f \mapsto f \mid\left(\begin{array}{ll}a & 0 \\ 0 & 1\end{array}\right)$ (where $a \in \mathbf{K}^{\times}, a \gg 0$ ) so that the range of the operators is one of the component spaces of $\mathscr{M}_{k}(\mathscr{N}, \chi)$; this isomorphism depends only on the ideal $a \mathscr{O}$ when $f \mid\left(\begin{array}{ll}u & 0 \\ 0 & 1\end{array}\right)=f$ for all totally positive units $u$.

Now we can prove

Lemma 4.2. Suppose $\mathscr{P}$ is a prime ideal not dividing $2 S(L)$ such that $L / \mathscr{P} L$ is hyperbolic; let $K$ be a $\mathscr{P}$-sublattice of $L$. Then

$$
\theta(\operatorname{gen} L, \tau) \mid T(\mathscr{P})=N(\mathscr{P})^{k / 2}\left(N(\mathscr{P})^{k-1}+1\right) \theta(\text { gen } K, \tau) .
$$

Proof. Let $L_{1}, \ldots, L_{t}$ and $K_{1}, \ldots, K_{s}$ be full sets of representatives for the classes in gen $L$ and gen $K$ (respectively); let $f_{i j}$ be the number of isometries $\sigma$ of $V$ which map $K_{j}$ into $L_{i}$ such that $\mathscr{P} L_{i} \subset \sigma\left(K_{j}\right)$. Then one has thie following easily verified facts:

(1) $f_{i j} \neq 0$ if and only if $K_{j}$ is isometric to a $\mathscr{P}$-sublattice of $L_{i}$.

(2) $1 /\left|O\left(K_{j}\right)\right| \cdot f_{i j}$ is the number of $\mathscr{P}$-sublattices of $L_{i}$ which are isometric to $K_{j}$. 
(3) $\left|O\left(L_{i}\right)\right| /\left|O\left(K_{j}\right)\right| \cdot f_{i j}$ is the number of isometries $\sigma$ of $V$ which map (4) $\mathscr{P} L_{i}$ into $K_{j}$ such that $\mathscr{P} K_{j} \subset \sigma\left(\mathscr{P} L_{i}\right)$.

$$
\sum_{j} \frac{1}{\left|O\left(K_{j}\right)\right|} \cdot f_{i j}=\left(N(\mathscr{P})^{k-1}+1\right) \lambda=\sum_{i} \frac{1}{\left|O\left(L_{i}\right)\right|} \cdot f_{i j}
$$

where $\lambda$ is as in Theorem 4.1. (To verify (4), see Proposition 7.2 of [11].)

The lemma now follows easily.

Let us assume that $\Gamma_{0}(S(L), N(L))=\Gamma_{\lambda \eta}(S(L))$ for some $\lambda$ and $\eta$ (notice that we can always scale $L$ to effect this). Our intention is to form a weighted average of theta series to obtain a $\mathscr{T}_{0}$-eigenform in $\mathscr{M}_{k}\left(\Gamma_{\lambda \eta}(S(L)), \chi_{L}\right)$ which we will then use to construct a $\mathscr{T}$-eigenform in $\mathscr{M}_{k}\left(S(L), \chi_{L}\right)$.

We first observe that the sum

$$
\sum_{u} \theta\left(\operatorname{gen} L^{u}, \tau\right)
$$

is an element of $\mathscr{M}_{k}\left(\Gamma_{\lambda \eta}(S(L)), \chi_{L}\right)$ (where the sum is over totally positive units $u$ which give us distinct genera gen $L^{u}$ ); however, this sum is not necessarily a $\mathscr{T}_{0}$-eigenform. The preceding lemma together with Proposition 6.1 of [11] shows that for $\chi_{L}=1, \alpha \gg 0, I$ a fractional ideal (relatively prime to $S(L)$ ), and $\mathscr{P}_{1}, \ldots, \mathscr{P}_{r}$ prime ideals not dividing $2 S(L)$ with $\alpha \mathscr{J}^{2} \mathscr{P}_{1} \ldots \mathscr{P}_{r}=\mathscr{O}$, we have

$$
\sum_{u} \theta\left(\operatorname{gen} L^{u}, \tau\right) \mid T_{0}\left(\mathscr{P}_{1} \ldots \mathscr{P}_{r}\right) S(\mathscr{I})=c \cdot \sum_{u} \theta\left(\operatorname{gen} \mathscr{I} K_{r}^{u \alpha}, \tau\right)
$$

where $c \neq 0, K_{j}$ is a $\mathscr{P}$-sublattice of $K_{j-1}, K_{0}=L$, and $K_{r}^{\alpha}$ is the lattice $K_{r}$ scaled by $\alpha$. Although $N\left(\mathscr{I} K_{r}^{u \alpha}\right)=N(L)$ and $S\left(\mathscr{I} K_{r}^{u \alpha}\right)=S(L)$, we may not have $\mathscr{I} K_{r}^{u \alpha} \in \operatorname{gen} L^{v}$ for any $v \in \mathscr{O}^{\times}, v \gg 0$ (consider, for instance, the case where $\mathscr{P}$ is nondyadic, $\alpha$ is not a square in $\mathscr{O}_{\mathscr{P}}$, and $L_{\mathscr{P}}$ has some Jordan components of odd rank). So to obtain a $\mathscr{T}_{0}$-eigenform we need to consider more lattices than those in gen $L$; thus we make the following

Definition. Let $L$ be a lattice on a quadratic space $V$, and let $\alpha \gg 0$. A lattice $K$ on $V^{\alpha}$ is in the family of $L$, fam $L$, if for every prime ideal $\mathscr{P}$ there is a unit $u_{\mathscr{P}} \in \mathscr{O}_{\mathscr{P}}$ such that $K_{\mathscr{P}}^{u_{\mathscr{P}}} \simeq L_{\mathscr{P}}$.

Notice that the family of a lattice $L$ can be partitioned into a finite number of genera, since $L_{\mathscr{P}}^{u_{\mathscr{P}}} \simeq L_{\mathscr{P}}$ whenever $\mathscr{P} \nmid 2 S(L)$ and $u_{\mathscr{P}} \in \mathscr{O}_{\mathscr{P}}^{\times}$. (Recall also that there are, up to isometry, only a finite number of spaces $V^{\alpha}$.) Note that in the special case where $V$ is a quadratic space over $\mathbb{Q}$ and $L$ is a unimodular lattice with $N(L) \subseteq \mathbb{Z}$, we have fam $L=\operatorname{gen} L$ (see Chapter 6 of [5] and note that the rank of such a lattice $L$ must be a multiple of 8 ).

So with $L$ and $K=\mathscr{I} K_{r}^{u \alpha}$ as in the discussion preceding this definition, we see that $K \in$ fam $L$ where we take $u_{\mathscr{D}}=1$ if $\mathscr{P} \nmid 2 S(L)$, and $u_{\mathscr{P}}=\alpha^{-1} \pi^{2}$ if 
$\mathscr{P} \mid 2 S(L)$ with $\pi \mathscr{O}_{\mathscr{P}}=\mathscr{J}^{-1} \mathscr{O}_{\mathscr{P}}$. Now we have

Theorem 4.3. Let $L$ be as above. Define

$$
\theta(\operatorname{fam} L, \tau)=\sum_{L^{\prime}} \frac{1}{\left|O\left(L^{\prime}\right)\right|} \theta\left(L^{\prime}, \tau\right)
$$

where the sum runs over a complete set of representatives of the isometry classes in fam $L$. Then $\theta(\operatorname{fam} L, \tau)$ is a nonzero element of $\mathscr{M}_{k}\left(S(L), \chi_{L}\right)$; if $\chi_{L}=1$ then $\theta(\operatorname{fam} L, \tau)$ is a $\mathscr{T}_{0}$-eigenform.

Note that when $\chi_{L}=1$, Lemma 4.2 together with Proposition 6.1 of [11] allows us to compute the eigenvalues for $\theta(\operatorname{fam} L, \tau)$; note in particular that these eigenvalues are nonzero. Now, applying Theorem 2.1 , we obtain

Corollary 4.4. Let the notation be as in Theorem 4.3, and suppose $\chi_{L}=1$ and that $\Gamma_{\lambda \eta}(S(L))=\Gamma_{0}(S(L), N(L))$. Then we can lift $\theta(\operatorname{fam} L, \tau)$ to $h$ linearly independent $\mathscr{T}$-eigenforms of $\mathscr{M}_{k}\left(S(L), \chi_{L}\right)$. Let $\mathscr{P}$ be a prime such that $\mathscr{P} \nmid 2 S(L)$ and $\mathscr{J}_{\mu} \mathscr{J}_{\sigma}^{2} \sim \mathscr{P}_{\lambda} \mathscr{I}_{\eta}^{2}$; then the $\mu, \eta$-component of any lift of $\theta(\operatorname{fam} L, \tau)$ is $c_{\mu \sigma} \cdot \theta(\operatorname{fam} K, \tau)$ where $c_{\mu \sigma} \neq 0$ and $K$ is a $\mathscr{P}$-sublattice of $L$.

\section{REFERENCES}

1. M. Eichler, On theta functions of real algebraic number fields, Acta Arith. 33 (1977), 269-292.

2. E. Hecke, Lectures on the theory of algebraic numbers, Springer-Verlag, New York, 1981.

3. W. Li, Newforms and functional equations, Math. Ann. 212 (1975), 285-315.

4. A. Ogg, Modular forms and Dirichlet series, Benjamin, New York, 1969.

5. O. T. O'Meara, Introduction to quadratic forms, Springer-Verlag, New York, 1973.

6. T. R. Shemanske and L. H. Walling, Twists of Hilbert modular forms, submitted 1990.

7. G. Shimura, The special values of the zeta functions associated with Hilbert modular forms, Duke Math. J. 45 (1978), 637-679.

8. Ann. of Math. 111 (1980), 313-375.

9. C. L. Siegel, Über die analytische Theorie der quadratischen Formen, Gesammelte Abhandlungen, Springer-Verlag, New York, 1966, pp. 326-405.

10. _ Über die analytische Theorie der quadratischen Formen III, Gesammelte Abhandlungen, Springer-Verlag, New York, 1966, pp. 469-548.

11. L. H. Walling, Hecke operators on theta series attached to lattices of arbitrary rank, Acta Arith. 54 (1990), 213-240.

Department of Mathematics, University of Colorado, Boulder, Colorado 80309 